\title{
Quantification of porcine circovirus type 2 (PCV-2) within- and between-pen transmission in pigs
}

\author{
Mathieu ANDRAUd ${ }^{1 *}$, Béatrice Grasland ${ }^{1}$, Benoît DurAND $^{2}$, \\ Roland CARIOLET ${ }^{1}$, André JeStin ${ }^{1}$, François MADEC ${ }^{1}$, Nicolas Rose ${ }^{1}$ \\ ${ }^{1}$ AFSSA-site de Ploufragan, B.P. 53, 22440 Ploufragan, France \\ 2 AFSSA-site de Maisons-Alfort, 23 avenue du Général de Gaulle, Maisons Alfort Cedex, 94706, France
}

(Received 19 October 2007; accepted 10 April 2008)

\begin{abstract}
PCV-2 within- and between-pen transmission was quantified by estimating the daily transmission rate $\beta$ and the basic reproduction ratio $\left(R_{0}\right)$ using a stochastic SEIR (Susceptible, Exposed, Infectious, Removed) model fitted on experimental data. Within-pen transmission was quantified by using four groups of eight SPF (specific pathogen-free) pigs (four infected and four susceptible pigs having direct contact). Between-pen transmission was studied in two groups of 16 SPF pigs (eight infected and eight susceptible pigs having indirect contact $(10 \mathrm{~cm}$ distance)). Pigs were monitored twice a week (blood samples) and were tested for PCV-2 antibodies (ELISA test) and viral genome load in sera (real-time PCR). Transmission parameters $\beta_{\text {within }}$ and $\beta_{\text {between }}$ were estimated using a maximum likelihood method and the duration of infectiousness, to compute $R_{0}$, was estimated with a parametric survival model. Different assumptions were made to determine the end of infectiousness (seroconversion, seroconversion and decline in viral genome load, permanent infectiousness). $R_{0 \text { [within] }}(8.9$ (5.1-15.4)) was greater when the end of infectiousness was assumed to be related to both seroconversion and a decline of PCV-2 genome load in sera (average duration of infectiousness $=32$ days) compared with only seroconversion as the indicator of recovery $\left(R_{0[\text { within] }}=5.5(3.3-9.0)\right)$. Whatever the assumption, between-pen $R_{0}(0.58(0.23-1.47))$ was always significantly lower than within-pen $R_{0}$. Only $\beta_{\text {within }}$ was sensitive to the assumption on end of infectiousness and decreased with increasing duration of infectiousness. These results showed that PCV-2 transmission is influenced by contact structure that appears worth being taken into account in an epidemic model.
\end{abstract}

PCV-2 / pigs / transmission / modelling

\section{INTRODUCTION}

Porcine circovirus type $2(\mathrm{PCV}-2)$ is a small single-stranded DNA virus known to be the aetiological agent of Post-weaning Multisystemic Wasting Syndrome (PMWS) in pigs $[3,28]$. This disease is of great economical importance in the majority of pig-producing countries throughout the world because of increased mortality in severely affected farms and growth retardation in cases of subclinical infection $[17,28]$.

* Corresponding author: m.andraud@afssa.fr
The main target of the virus is the immune system and it leads to a severe immune suppression resulting in an increased susceptibility to other viral or bacterial infections [29]. The pathogenesis of PMWS is not well known. Many studies have shown that PCV-2 has widely spread in the pig population since decades, before the first descriptions of the syndrome [18,24]. Maternal immunity is protective and pigs get the infection mainly at the beginning of the growing phase [20, 21, 23]. PCV-2 is known to be shed mainly in faeces and oro-nasal fluids [30]. Direct contact between pigs is supposed to be the main route 
of transmission of PCV-2. Airborne transmission on a short distance is likely to occur but to the best of our knowledge, it has never been demonstrated in an experimental setting.

Several studies have demonstrated the quantitative feature of this disease because of the strong relationship between lesion severity and the amount of virus within the affected organs [15]. Moreover, PMWS hazard is highly related to the time to infection: the earlier, the higher the risk [26]. Segregation of piglets in small groups was also found protective [25]. Both observations suggest that early segregation of piglets from different infectious statuses might decrease the force of infection within the room and postpone the infection for susceptible pigs to a later stage, when they are less likely to express the disease.

Several epidemiological studies have been carried out to identify risk factors for PMWS in pig farms ${ }^{1}[16,25,33]$. These observational studies in field conditions often reveal PMWS occurrence to be associated with husbandry practices and within-herd prevalence of PCV-2 in specific age categories. Transmission experiments can indicate how separation of infected and susceptible pigs might contribute to lower prevalence and later infection, to reduce PMWS occurrence. Such information could be further used in a modelling approach for analysis and extrapolation to bigger groups (whole population of a pig farm).

Transmission experiments in comparison with field studies offer a controlled environment in which a single factor can be investigated. As a consequence, more insight can be obtained into causative mechanisms underlying the transmission dynamics of the pathogen. In transmission experiments, pure infections can be reproduced without interference owing to other infectious agents. In addition, using SPF (specific pathogen-free) PCV-2-negative animals avoids confusion with aspects related to PCV-2 maternal antibodies. Data produced

${ }^{1}$ Enoe C., Vigre H., Nielsen E.O., Botner A., Bille-Hansen V., Jorsal S.E., Baekbo P., A Danish case-control study on risk factors for PMWS Biosecurity in the herd, 19th IPVS Congress (2006) 1:O.27-03.

Page 2 of 13 (page number not for citation purpose) from transmission experiments can be used to parameterise an epidemiological model which is then used to estimate specific biologically interpretable parameters, and to test hypotheses [31].

The aim of this study was to estimate transmission parameters, based on experimental data, that could be further used in an infectious model for PCV-2 within-herd infection. Such parameters are of great importance to properly describe the within-herd dynamics of PCV-2 infection and represent the heterogeneity in contact structure in the modelling process. Within- and between-pen transmissions were studied estimating the transmission parameter $\beta$ (mean number of secondary infections caused by a single infectious animal per unit of time) and the basic reproduction ratio $R_{0}$ (mean number of secondary infections occurring from a single infected animal during its infectious period in a large susceptible population). Transmission parameters $\beta_{\text {within }}$ and $\beta_{\text {between }}$ were estimated using a maximum likelihood method and the duration of infectiousness for $R_{0}$ computing was estimated with a parametric survival model.

\section{MATERIALS AND METHODS}

\subsection{Experimental design}

\subsubsection{Animals}

Seventy-two Large-White SPF pigs aged 28 days at the start of the experiment were used. They were derived from the AFSSA (Agence Française de Sécurité Sanitaire des Aliments) SPF herd ${ }^{2}$.

\subsubsection{Trial description}

The experiment was conducted in our air-filtered level 3 biosecurity-facilities (Fig. 1). Four independent rooms were used (R1 to R4), each of them including two pens. Rooms 1 and 2 were used to assess between pen transmission. The two adjacent pens, located $10 \mathrm{~cm}$ apart, housed respectively

\footnotetext{
${ }^{2}$ Cariolet R., Le Diguerher G., Ecobichon P., Julou P., Jolly J.P., Madec F., Production of long term, low-cost specific pathogen free pigs, Symposium of the International Society for Animal Hygiene, St Malo, France (2004) 149.
} 


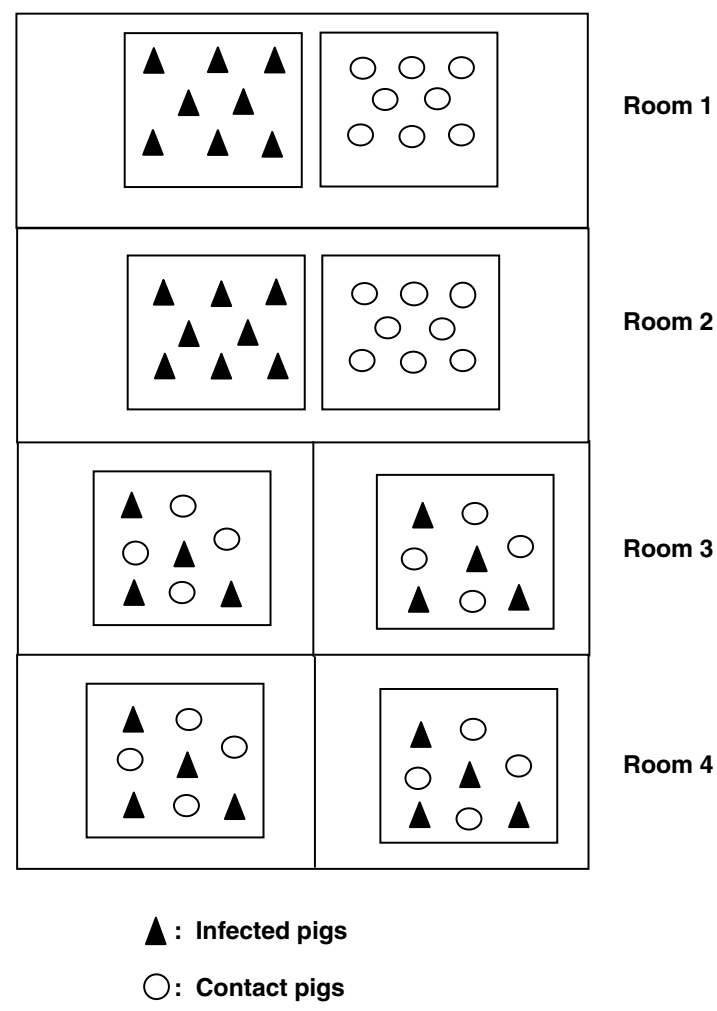

Controls

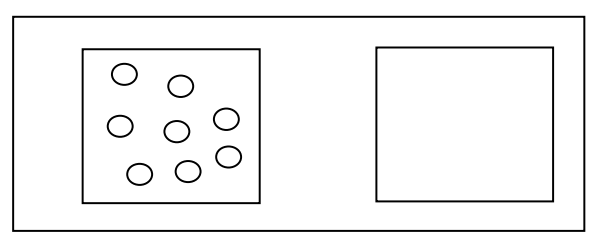

Figure 1. Experimental design for quantification of within- and between-pen transmission of PCV-2.

eight inoculated and eight contact pigs. The two pens had open partitions but the pigs could not be in direct physical contact. Rooms 3 and 4 were used to assess within-pen transmission. In a room, each pen was conducted separately (solid partition between pens). In each pen, four inoculated and four contact pigs were mingled. In addition, a pen located in a separate room housed eight SPF pigs used as negative controls. Each pen was submitted to a specific air-extraction system to prevent airborne transmission of the virus from one pen to another. Solid plastic partitions between pens in rooms 3 and 4 prevented transmission of infectious material from one pen to another. Moreover, strict biosecurity measures were carried out when entering the different rooms (shower and specific clothes in each room) and between pens (change of boots and clothes). In rooms 1 and 2, animal care systematically began with contact groups and then inoculated ones to prevent from human-based transmission between pens. The experiment was performed in accordance with EU and French regulations on animal welfare during experimentations.

\subsubsection{Infection}

Infected pigs were inoculated at day 0 with $6 \mathrm{~mL}(5 \mathrm{~mL}$ intratracheal $+1 \mathrm{~mL}$ intramuscular) of a PCV-2 suspension $\left(10^{5} \mathrm{TCID}_{50} / \mathrm{mL}\right)$ as described previously [1]. Contact pigs were introduced for indirect or direct contact at day 1.

\subsubsection{PCV-2 monitoring}

The PCV-2 statuses of the inoculated and contact pigs were monitored once and twice a week respectively until 42 days post-inoculation (dpi) using real-time PCR [9] in sera to assess the PCV-2 genome load. Pigs were declared as infected as soon as PCV-2 genome load was detected in the serum. In addition, PCV-2 antibodies were monitored weekly with an ELISA test [5].

\subsubsection{End of the experiment}

Four pigs per pen were killed at $35 \mathrm{dpi}$ in order to maintain the required space per pig. In rooms 3 and 4, two inoculated and two contact (page number not for citation purpose) Page 3 of 13 
pigs were randomly selected. The remaining pigs were killed at 42 dpi. Euthanasia was carried out by anaesthesia with intravenous injection of $1 \mathrm{~g} / 50 \mathrm{~kg}$ liveweight of Nesdonal ${ }^{\circledR}$ (Merial, Lyon, France) followed by exsanguination. At necropsy, immediately after killing, the following organs were examined macroscopically: lung, tonsil, heart, kidney, thymus, ileum, and lymph nodes (inguinal, mesenteric, tracheo-bronchial). If macroscopic lesions were suspected, samples from the tissues were taken for histopathological examination.

Histological examination was carried out on the different tissues after fixation with $10 \%$ formaldehyde (Sigma-Aldrich, St Louis, MO, USA). Sections $(8 \mathrm{~mm})$ were stained with haemalum-eosinsafranin (Sigma-Aldrich) and observed under a light microscope. In-situ hybridisation was performed on sections $(4 \mathrm{~mm})$ of tissues which were washed, heated to $40^{\circ} \mathrm{C}$, deproteinised, and then hybridised at $55^{\circ} \mathrm{C}$ with the PCV-2-specific probe $5^{\prime}$ CCGTTGTCCCTGAGATCTA 3' conjugated at both ends with digoxigenin (Sigma-Aldrich). After three washings and a single incubation at $40^{\circ} \mathrm{C}$, a biotinylated mouse monoclonal antibody to digoxigenin (Ventana Medical, Strasbourg, France), a peroxidase-avidin complex (Sigma-Aldrich) and 3-amino-9-ethyl-carbazole (Serotec, Oxford, UK) were applied in succession. The slides were finally counterstained with haemalum and blueing reagent (Ventana Medical).

\subsection{Serology}

Serum samples were tested for PCV-2 antibodies by an ELISA based on the recognition of a recombinant PCV-2 capsid protein/GST-fused protein and a GST (Glutathione S-transferase) protein [5]. Samples with an OD (Optical Density) ratio higher than 1.5 were considered positive for PCV-2 antibodies (sensitivity: 0.98 and specificity: 0.95, taking IPMA (Immunoperoxidase Monolayer Assay) as a reference).

\subsection{Quantification of PCV-2 genomes by real-time Polymerase Chain Reaction (PCR)}

DNA was extracted from $200 \mu \mathrm{L}$ of each tested serum using the Wizard SV96 genomic DNA purification system (Promega, Madison, Wisconsin, USA) according to the manufacturer's instructions. Elution was performed with $250 \mu \mathrm{L}$ of sterile $\mathrm{H}_{2} \mathrm{O}$ and $5 \mu \mathrm{L}$ of this extraction corresponding to $4 \mu \mathrm{L}$ of serum, were used as template for PCV-2 TaqMan PCR. Controls during DNA extraction were carried out by replacing serum with PBS (Qbiogene, Illkirch, France), every five samples in order to check any PCV-2 contamination.

The number of PCV-2 genome copies was assessed by a real-time PCR based on TaqMan technology as described previously [9]. For each PCR run, a positive control obtained from lymph node tissue from PCV-2-infected pigs, was included. Four negative controls were also introduced by replacing the DNA sample by purified water, with two negative controls close to the positive control and the other two negative controls at the end of the plate. The lack of amplification was also checked by PCV-2quantitative PCR with the negative controls of the DNA extraction. Samples were processed in duplicate. The limit detection of the technique is $<10^{3} \mathrm{PCV}-2$ copies $/ \mathrm{mL}$.

The mean log-number of PCV-2 copies per $\mathrm{mL}$ was compared in contact pigs between direct and indirect contact groups at different dates $(31,35,38$ and 42 days) post-infection using ANOVA (GLM procedure, [27]) and testing for a group, room and interaction group-room effects.

\subsection{Estimation of the basic reproduction ratio $\left(R_{0}\right)$}

We used the method described previously [12], based on maximum likelihood estimates of transmission parameters. This algorithm relies on a SEIR (Susceptible, Exposed, Infectious, Recovered) model with a constant latent period $\mathrm{E}$ of six days (infected but not infectious), according to the results of an immunoperoxydase monolayer assay (IPMA) [9] on sera taken from inoculated pigs at 4 and 7 dpi (data not shown). Briefly, sera were tested for infective particles in cell cultures (PK15 monolayer). Only few infected cells could be detected with sera taken at $4 \mathrm{dpi}$. Conversely, at $7 \mathrm{dpi}$, all sera tested positive for infective virus with an infective titre being higher than $10^{2.5} \mathrm{TCID}_{50} / \mathrm{mL}$ and leading to an assumption of six days for the duration of latency.

In this model, the rate by which susceptible pigs were infected is $\beta S I / N$, the rate by which infective pigs recovered is $\sigma \mathrm{I}$, where $\beta$ and $\sigma$ are the transmission and recovery parameters respectively. The recovery parameter $\sigma$ is defined as the inverse 
of the mean duration of the infectious period. In this particular situation of a closed SEIR model $R_{0}=\frac{\beta}{\sigma}, R_{0}$ being the mean number of secondary infections occurring from a single infected animal during its infectious period in a large susceptible population.

From the model it follows that the probability $q$ that a single susceptible animal escapes infection in a time interval of duration $d$ is equal to $q=$ $\exp \left(-d\left(\beta_{w} \pi_{w}+\beta_{b} \pi_{b}\right)\right)$. In this equation, $\pi_{w}$ and $\pi_{b}$ are the proportions of infectious animals within the same pen and in contact pens, respectively, and $\beta_{w}$ and $\beta_{b}$ are the transmission parameters within and between pens, respectively. $p=1-q$ is then the probability for a susceptible individual to be infected and the number of new infections follows a Binomial distribution $p \approx \operatorname{Bin}(S, p)$ with $S$ the number of susceptible animals in contact. The loglikelihood for this Binomial distribution is

$$
\begin{aligned}
\log L\left(\beta_{w}, \beta_{b}\right)= & \sum_{i}\left[C_{i} \log \left(e^{d_{i}\left(\beta_{w} \pi_{w}^{i}+\beta_{b} \pi_{b}^{i}\right)}-1\right)\right. \\
& \left.-S_{i}\left(d_{i}\left(\beta_{w} \pi_{w}^{i}+\beta_{b} \pi_{b}^{i}\right)\right)\right]
\end{aligned}
$$

with $S_{i}$ and $C_{i}$ the number of susceptible pigs and cases at each time interval $i$ respectively and where $\log \left(\begin{array}{l}S_{i} \\ C_{i}\end{array}\right)$ is omitted because it plays no role in the calculations. Both parameters $\beta$ were estimated using maximisation of the log-likelihood with the $n l m$ function in R software (Version 2.6.0., [11]). Confidence intervals were determined using the inverse of the Hessian matrix (variance/covariance matrix) of parameter estimates, which is also provided by the $n l m$ function in $\mathrm{R}$ software [11]. The duration of infectiousness was estimated from this experiment using a parametric survival model with an exponential distribution (function SURVREG in $\mathrm{R}$ software [11]) and considering different assumptions on the termination of infectiousness: (1) pigs were considered as 'recovered' as soon as they seroconverted (Assumption (1)); (2) pigs were considered as 'recovered' when they seroconverted and evidenced a decline in the PCV-2 genome load in sera (Assumption (2)); (3) infectiousness lasted until the end of the experiment (Assumption (3)). For the assumption (2), the time of decline in PCV-2 genome load was determined as follows: the day when the maximum genome load was attained was taken as reference. Then, the dpi declared as being related to a decline in genome load was taken as the following next sampling day, corresponding to a lower value compared with the reference (peak), together with an already established serological response. In case of no evidence of decreasing trend, the last sampling date was taken.

\section{RESULTS}

\subsection{PCV-2 genome load and time to seroconversion in inoculated and contact pigs}

In inoculated pigs, PCV-2 genome load in sera increased from 0 to $7 \mathrm{dpi}$, reaching a peak $\left(10^{6}\right.$ copies $/ \mathrm{mL}$ on average $)$ at $14 \mathrm{dpi}$ to further slightly decrease until the end of the experiment ( $42 \mathrm{dpi})$. Seroconversion was observed between 14 and $21 \mathrm{dpi}$ for the majority of the pigs. No difference could be evidenced between inoculated pigs from the within- or between-pen transmission groups for both genome loads and serological results (Figs. 2 and 3).

The first contact pig detected as infected was found at 17 days post-contact, in the indirect contact group (room 2). The other first infected pigs were detected at 21 days post-contact or later. At 28 days post-contact 15 out of 16 pigs were already infected with a high genome load (between $10^{5}$ and $10^{7}$ PCV-2 copies $/ \mathrm{mL}$ ) in the direct contact group whereas only 5 were detected in the indirect contact group (Figs. 2 and 3). PCV-2 genome load in sera increased within a week from the first detection day for the direct contact group whereas the increase was more progressive in the indirect contact group with mean PCV-2 genome copies/mL significantly lower than in the direct contact group at $31(p<0.001)$ and $35(p=0.05)$ days post-contact. There was no further difference at 38 and 42 days. No significant difference was found within groups and between rooms (Figs. 2 and 3 ).

At the end of the experiment $(35 \mathrm{dpi})$ the magnitude of genome load in contact pigs was found similar to the one in inoculated pigs at 14 dpi.

The eight controls remained PCR negative in sera and seronegative until the end of the experiment. 

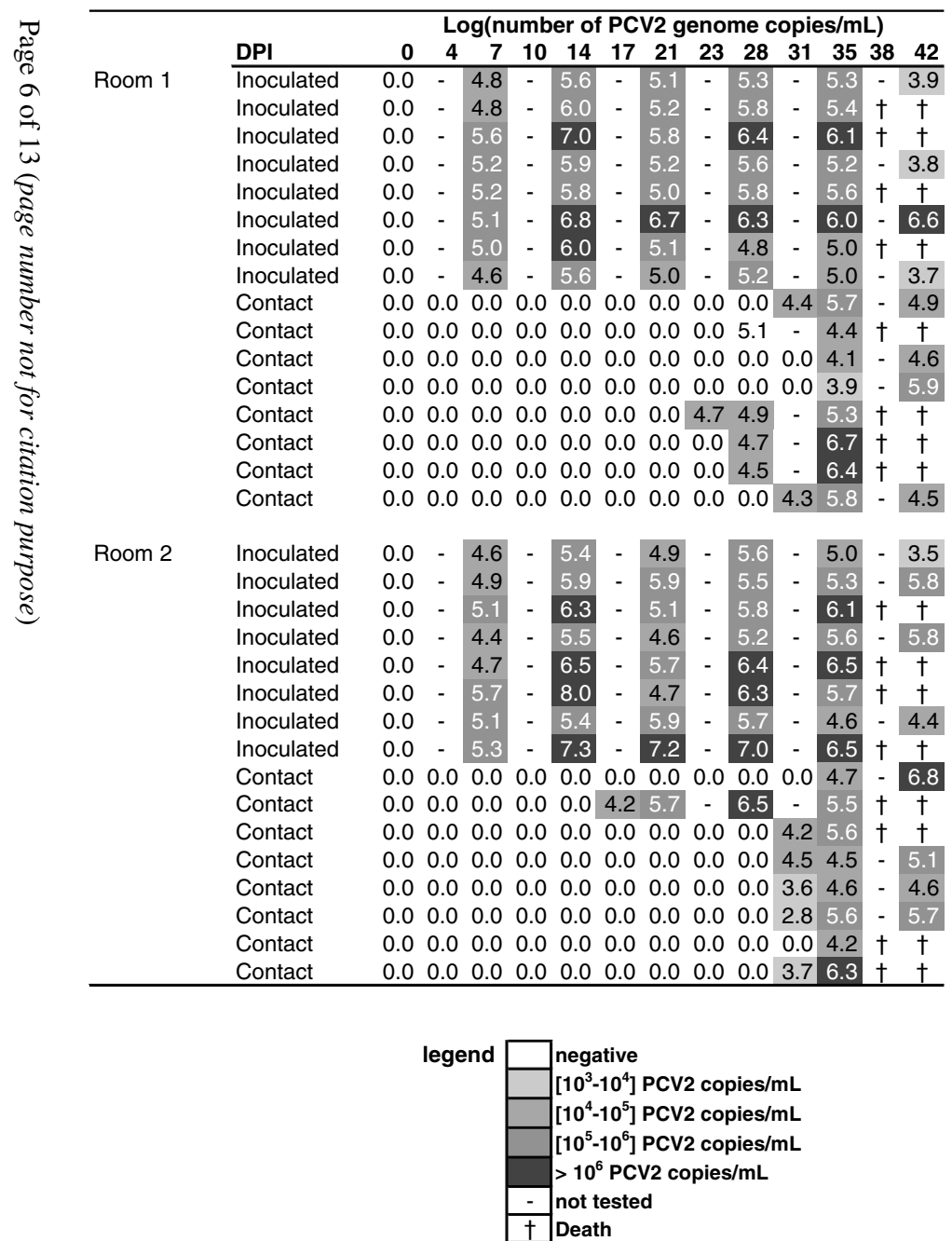

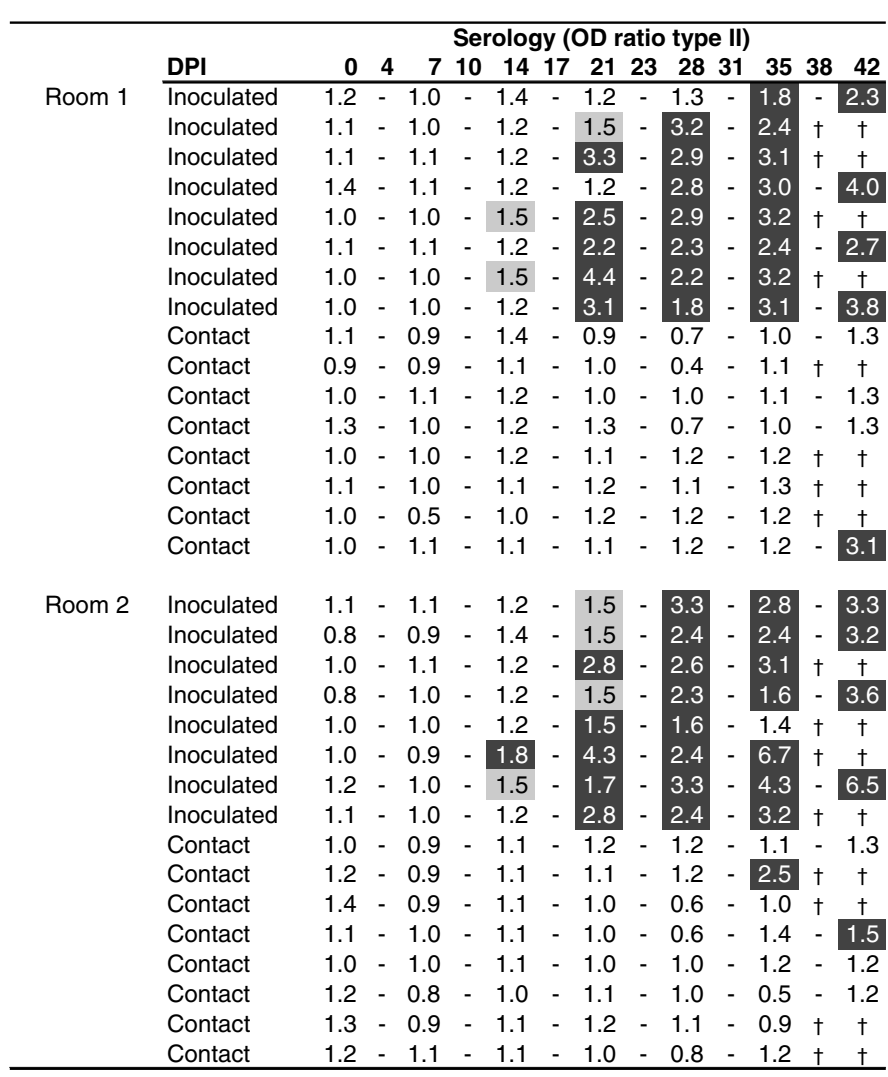

legend

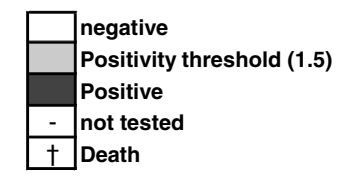

Figure 2. Kinetics of infection in pigs for indirect contact (between-pen transmission) group: log-number of PCV-2 genome load in sera (A) and serological results (B). 


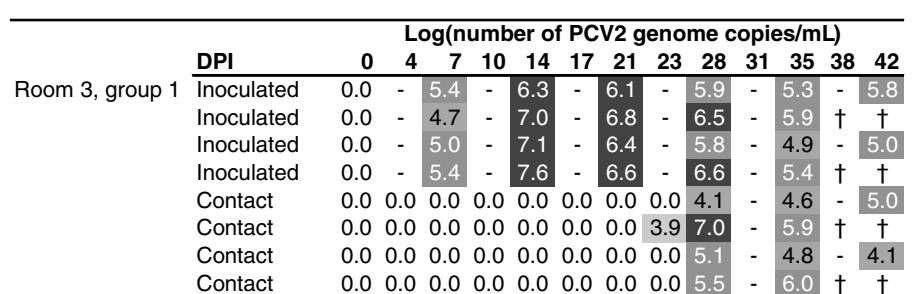

Room 3, group 2 Inoculated $0.0-53-6.2-6.1-54=-5.10$

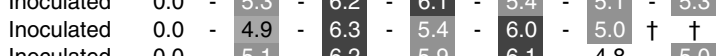

Inoculated $0.0-5.1-6.2-5.9-6.1-4.8-5.0$

Inoculated $0.0-4.9-6.3-5.7-6.1-5.3+\dagger$

Contact

Contact

Contact

$\begin{array}{llllllllll}0.0 & 0.0 & 0.0 & 0.0 & 0.0 & 0.0 & 0.0 & 3.1 & 7.2 \\ 0 & 0 & 0 & 0 & 0 & 0 & 0 & 0 & 0 & 0\end{array}$

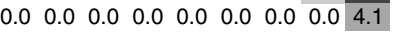

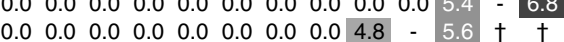

Room 4, group 1 Inoculated

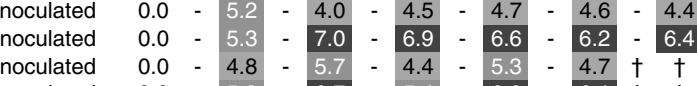

Inoculated $0.0-4.8-5.7-4.4-5.3-4.7 \dagger+$

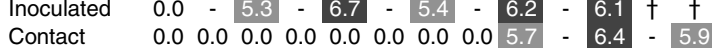

$\begin{array}{llllllllll}0.0 & 0.0 & 0.0 & 0.0 & 0.0 & 0.0 & 0.0 & 3.9 & 6.2\end{array}$

Contact $\quad \begin{array}{lllllllll}0.0 & 0.0 & 0.0 & 0.0 & 0.0 & 0.0 & 0.0 & 2.9 & 6.5\end{array}$

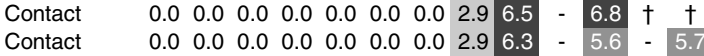

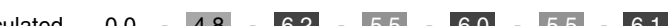

Inoculated

Inoculated

Inoculated

Contac
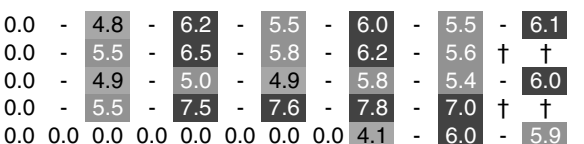

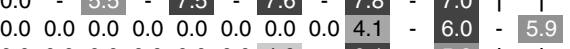

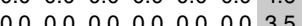

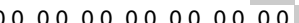

legend $\square$ negative

$-\left[\begin{array}{l}\text { negative } \\ {\left[10^{3}-10^{4}\right] \text { PCV2 copies } / \mathrm{mL}} \\ {\left[10^{4}-10^{5}\right] \text { PCV2 copies } / \mathrm{mL}}\end{array}\right.$

$\left[10^{5}-10^{6}\right]$ PCV2 copies $/ \mathrm{mL}$

$>10^{6} \mathrm{PCV} 2$ copies $/ \mathrm{mL}$

not tested

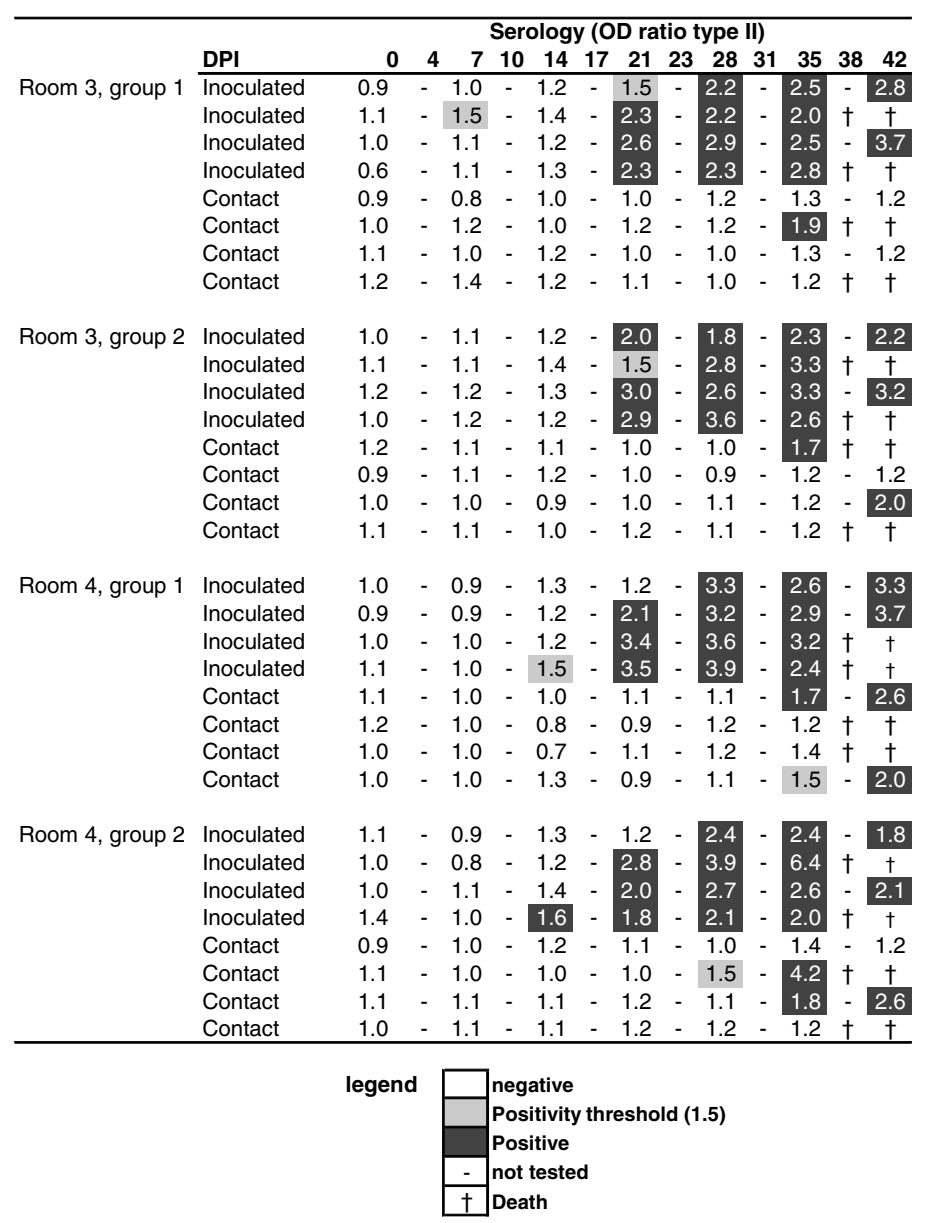

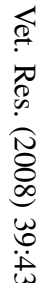

results (B) 
Table I. Estimation of parameters with $95 \%$ confidence intervals according to the different assumptions on the duration of infectiousness with $\beta$ the mean number of secondary infections caused by a single infectious animal per unit of time, $1 / \sigma$ the duration of the infectious period and $R_{0}$, the basic reproduction ratio (mean number of secondary infections occurring from a single infected animal during its infectious period in a large susceptible population).

\begin{tabular}{|c|c|c|c|c|c|c|}
\hline & \multicolumn{3}{|l|}{ Within-pen } & \multicolumn{3}{|l|}{ Between-pen } \\
\hline & Assumption $(1)^{\mathrm{a}}$ & Assumption $(2)^{\mathrm{b}}$ & Assumption $(3)^{\mathrm{c}}$ & Assumption $(1)^{\mathrm{a}}$ & Assumption $(2)^{\mathrm{b}}$ & Assumption (3) \\
\hline$\beta$ & $0.31(0.20-0.45)$ & $0.28(0.18-0.42)$ & $0.23(0.14-0.36)$ & $0.03(0.01-0.08)$ & $0.04(0.02-0.08)$ & $0.04(0.02-0.09)$ \\
\hline$\sigma$ & $0.06(0.04-0.08)$ & $0.03(0.02-0.05)$ & - & $0.06(0.04-0.08)$ & $0.03(0.02-0.05)$ & - \\
\hline $1 / \sigma$ & $17.7(13.1-24)$ & $31.7(22-45.7)$ & - & $17.7(13.1-24)$ & $31.7(22-45.7)$ & - \\
\hline$R_{0}$ & $5.5(3.3-9.0)$ & $8.9(5.1-15.4)$ & - & $0.58(0.23-1.47)$ & $1.2(0.5-2.9)$ & - \\
\hline
\end{tabular}

${ }^{a}$ End of infectiousness when seroconverted.

${ }^{b}$ End of infectiousness if seroconversion AND decline of the PCV-2 genome load in serum.

${ }^{\mathrm{c}}$ Permanent infectiousness until the end of experiment (SEI model).

\subsection{Clinical and post-mortem findings}

Very mild symptoms were observed during the experiments with only pyrexia $\left(>40.5^{\circ} \mathrm{C}\right)$ in 3 inoculated pigs ( 2 from the direct contact group and 1 from the indirect contact group) between 15 and 20 dpi. No symptoms were observed in contact pigs. Lesions at necropsy were mild with only a slight enlargement of lymph nodes (inguinal, mesenteric, and tracheobronchial) mainly in inoculated pigs (6/16 and 5/16 for the direct and indirect contact groups respectively). In contact pigs only 6 had enlargement of lymph nodes $(5 / 16$ and $1 / 16$ in the direct and indirect contact groups respectively). From these organs, histopathology did not evidence typical histological lesions possibly related to PMWS. No lesion was observed in the control group.

\subsection{Parameter estimation}

$\beta$ estimate for within-pen transmission (0.31 (0.20-0.45)) was found to decrease when the duration of infectiousness increased according to the assumption on recovery (Fig. 4). $R_{0}(8.9$ (5.1-15.4)) was greater when the end of infectiousness was assumed to be related to both seroconversion and a decline of PCV-2 genome load in sera (average duration of infectiousness $=32$ days) compared with only seroconversion as the indicator of

Page 8 of 13 (page number not for citation purpose) recovery $\left(R_{0}=5.5 \quad(3.3-9.0)\right) \quad$ (Tab. I). $\beta$ estimates for between-pen transmission $(0.03$ (0.01-0.08)) were slightly modified according to the assumption on recovery (Fig. 4). Whatever the assumption, between-pen $R_{0}$ (0.58 (0.23-1.47)) was always significantly lower than within-pen $R_{0}$ (Tab. I).

\section{DISCUSSION}

Infection of SPF pigs by PCV-2 without immune enhancement [9, 14] or coinfection $[2,10,13]$ is known to lead to subclinically infected animals with mild symptoms and lesions as we observed in this study. However, even if no disease was reproduced as expected, the infection was successful and led to a similar genome load as observed in field conditions, in naturally sub-clinically infected animals $[8,30]$. The observed time to seroconversion in inoculated pigs and average number of PCV-2 DNA copies/mL in sera in our study were similar to a previous study with the same virus titre in the inoculum [8]. Similar genome loads were found in contact pigs that were mingled with inoculated ones showing that transmission was efficient even in subclinically infected pigs.

To start the infection chain in the different replicates of the experiment we used infected animals according to a previous experimental 


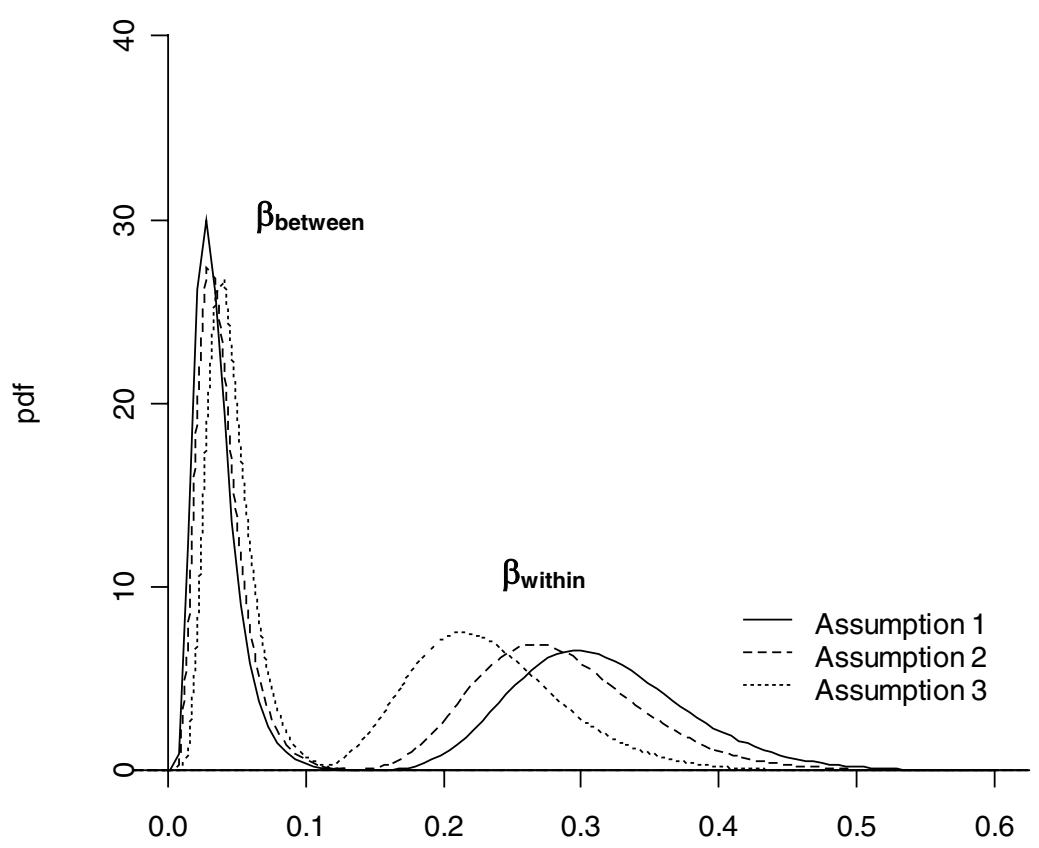

Figure 4. Probability density functions of the transmission rates $\beta$ according to the assumptions on end of infectiousness (Assumption (1) : end of infectiousness at seroconversion; Assumption (2): end of infectiousness when seroconverted and PCV-2 genome load in sera declined; Assumption (3): permanent infectiousness).

model for PMWS [1]. This rather 'unnatural' infection process including the inoculation via both the intratracheal and the intramuscular routes was set up in order to get a homogeneous and comparable population of infectious pigs whatever the group (within- or betweenpen transmission). Secondary infected pigs used as seeders to infect susceptible ones as in a transmission experiment with Actinobacillus pleuropneumoniae [32] would have been relevant because it would have reproduced, in a more natural way, infections occurring within a farm. However, the addition of such supplementary phases would have led to a necessary limitation of the duration of the contact trial per se because the required space per pig had to be maintained. The evolution of viremia (attested by PCV-2 genome load in sera) in inoculated pigs evidenced that there was an excellent homogeneity of the infectious status within groups. This observation is of a great importance because the model used for the estimation of parameters relies on a strong assumption of equal probability of infectious contacts between susceptible and infectious animals. The absence of a difference between groups (inoculated animals for within- of between-pen transmission) made the comparison possible between the estimated parameters.

The algorithm based on likelihood estimation method was carried out to estimate the transmission parameters in this experiment. This algorithm takes the time-course of the experimental epidemic into account: for each animal, an infection date and a period during which the animal has been infectious are computed [12,31].

Another well known algorithm ${ }^{3}[6]$ relies on the final size of epidemics, i.e. on the individual status of contact pigs (infected

\footnotetext{
${ }^{3}$ Kroese A.H., De Jong M.C., Design and analysis of transmission experiment, Annual meeting of the Society for Veterinary Epidemiology and Preventive Medicine (2001) 21-37.
} 
or not), assuming that the infection process has ended at the end of the experiment. In the present experiment, all contact pigs were infectious at the end of the experiment and no susceptible animal remained, so that the 'final size' algorithm could not give a point estimate of the parameter but only the lower bound of the confidence interval, the upper bound being infinity.

Taking the time course of the experimental infection into account with the maximum likelihood method was found to result in smaller confidence intervals for $R_{0}$ than the martingale method [12] because the data are more extensively used with a division of the viral infection process into intervals with known numbers of susceptible, infectious and recovered pigs. The underlying infectious SEIR model we used as described previously [12] relies on a frequency-dependent contact process i.e. the per capita contact rate is fixed and does not depend on the size of the population $[4,7,19], \beta S I / N$ being the rate by which new infections take place (number per unit of time). This assumption seems reasonable in the context of our experiment since (i) the area available for the pigs was limited because of the pen size and probability of contacts could be assumed to be uniform; and (ii) there was no change in the population size (no introduction and no mortality until the end of the experiment).

The definition of the infection status, susceptible or infectious, of an individual animal during the experiment is important for the estimation of the parameters. To the best of our knowledge, the duration of infectiousness for PCV-2 has not been studied. Only information is available on the duration of viremia or PCV-2 shedding but assessed with PCR or real-time PCR. In naturally infected pigs PCV-2 infection usually occurs after a decline of passive immunity between 72 and 84 days of age [20,23]. At 150 days of age, i.e. 50 days after the peak of viremia $50 \%$ of the pigs tested positive for DNA in serum [20] and only $10 \%$ were found positive 110 days after the peak of viremia [23]. Although PCR is a sensitive molecular diagnostic tool that can detect PCV-2 at low concentration in serum, it does not provide information on infective capability. The relationship between the production of neutralising antibodies (NA) and the absence of development of PMWS [22] and the negative correlation between NA titres and PCV-2 genome load in serum [8] are in favour of a protective effect of the NA response against PCV-2 replication. Neutralising antibodies were shown to increase by $21 \mathrm{dpi}^{4}$, slightly delayed in comparison with the results for IgG. The increase of NA was shown to coincide with the drop in viral load with less than $10^{3}$ DNA copies/mL serum after 50 days postinoculation [8]. Altogether, these data show that the average duration of infectiousness might be underestimated in our first assumption based on seroconversion only (ELISA test). However, the second assumption based on seroconversion with in addition a decline in PCV-2 genome load, is consistent with the data available on NA and the estimated duration of infectiousness (32 days) might be more realistic. However, the infectious status is characterised by the ability to infect a susceptible individual after a contact and neither indicators based on genome load in sera nor those based on DNA detection from nasal or rectal swabs can address the question. A proper way to estimate this parameter would be to carry out serial contacts with seeder pigs at different time periods after inoculation and check for infections in the different contact groups. This should be addressed in a further study.

From our results, the assumption on the end of infectiousness had a major impact on the estimate of $R_{0}$ (because it depends on the duration of infectiousness) but a rather moderate impact on $\beta$ parameters. Whatever the assumption, the difference between withinand between-pen transmissions was always significant.

\footnotetext{
${ }^{4}$ Opriessnig T., Madson D.M., Prickett J.R., Pogranichniy R.M., Meng X.J., Halbur P.G., Longterm effect of PCV2-infection and reinfection on PCV2-antibody and DNA profiles in pigs over time, 5th International Symposium on Emerging and Reemerging Pig Diseases (2007) 45.
} 
For within-pen transmission, the $\beta$ parameter was found to be higher in assumption (1) than in assumptions (2) and (3). In the third assumption (SEI model), the number of infectious animals involved in the process is strictly increasing with time, increasing the force of infection in the room as well. However, this number of infectious individuals is the only fluctuating parameter in the loglikelihood expression (all the others being fixed by experimental trial and observed results). Thus, the permanent increase in the number of infectious animals leads to a decrease in the estimated within-pen transmission rate to reach the observed total number of infections. In assumption (1), with a quite short duration of infectiousness, the transmission parameter was found to be higher with maximum likelihood estimation to get the same number of infected animals in the same time period. The estimates of between-pen transmission rate were not (or very slightly) modified according to the assumptions made on the end of infectiousness because this transmission is mainly important during the first steps of the infectious process, after which the within-pen transmission becomes far more efficient.

The difference between within- and between-pen transmission of PCV-2 suggests that the contact structure affects transmission of PCV-2 and that the slower spread between pens should be taken into account in a modelling approach of the within-herd dynamics of infection. The results on the between-pen transmission parameter clearly show that the average number of secondary infected animals per infectious animal and per time unit from one pen to another adjacent one is expected to be very low, which is an evidence of the expected efficacy of segregating pigs from different infectious statuses in different pens to postpone infections to a later stage. It also probably means that transmission between herds should be very limited without pig imports from an infected herd with infectious animals. Early PCV-2 infection in pigs $(<7$ weeks-old) has been found as a risk factor for PMWS [26] as well as mixing of animals with different statuses in large pens at weaning [25]. Hence segregating pigs of different statuses as long as possible might postpone transmission to a later stage when the infection is less likely to result in disease. This is consistent with the first field observations on PMWS in severely affected farms where a lot of mixings at different stages (cross-fostering in farrowing facilities, weaning, fattening sectors) were prominent in those farms with high mortality [17]. All the conditions were probably fulfilled to enhance PCV-2 (and certainly other pathogens) transmission.

The transmission rates that we estimated can be of use in a quantitative modelling approach to identify key management strategies that could be implemented to delay PCV-2 transmission and decrease PMWS incidence. Since a batch of fattening pigs can not be considered as homogeneous the results from the present study may be useful to take into account this heterogeneity in contact structure (pen clustering) to represent PCV-2 dynamics in a more realistic way. Such a quantitative modelling approach would be a useful tool to identify strategies based on husbandry practices to manage $\mathrm{PCV}-2$ infection and prevent massive infections occurring in the young age. The approach could also be used to study the implementation of vaccination since several vaccines (sow- or piglet-targeted) are and will be available in the next future. A further study is also needed on the characteristics of the infectious period (mean duration, shape of the distribution) since the behaviour and the prediction of the model would be highly dependent on these features.

Acknowledgements. The authors are grateful to Aurélie Oger, André Keranflec'h and Bruno Jan for their excellent technical assistance. We thank the referees for their helpful comments which largely contributed to improve this paper. This study was partly financed by AFSSA and INRA (project "transversalité AFSSA/INRA MODPROPAG").

\section{REFERENCES}

[1] Albina E., Truong C., Hutet E., Blanchard P., Cariolet R., L'Hospitalier R., et al., An experimental model for post-weaning multisystemic wasting syndrome (PMWS) in growing piglets, J. Comp. Pathol. (2001) 125:292-303. 
[2] Allan G.M., Kennedy S., Mcneilly F., Foster J.C., Ellis J., Krakowka S., et al., Experimental reproduction of severe wasting disease by co-infection of pigs with porcine circovirus and porcine parvovirus, J. Comp. Pathol. (1999) 121:1-11.

[3] Allan G.M., Ellis J.A., Porcine circoviruses: a review, J. Vet. Diagn. Invest. (2000) 12:3-14.

[4] Anderson R.M., May R.M., Infectious diseases of humans: dynamics and control, Oxford University press, 1992, p. 756.

[5] Blanchard P., Mahé D., Cariolet R., Truong C., Le Dimna M., Arnauld C., et al., An ORF2 proteinbased ELISA for porcine circovirus type 2 (PCV2) antibodies in post-weaning multisystemic wasting syndrome (PMWS), Vet. Microbiol. (2003) 94:183194.

[6] De Jong M.C., Kimman T.G., Experimental quantification of vaccine-induced reduction in virus transmission, Vaccine (1994) 12:761-766.

[7] De Jong M.C., Mathematical modelling in veterinary epidemiology: why model building is important, Prev. Vet. Med. (1995) 25:183-193.

[8] Fort M., Olvera A., Sibila M., Segalés J., Mateu E., Detection of neutralizing antibodies in postweaning multisystemic wasting syndrome (PMWS)affected and non-affected pigs, Vet. Microbiol. (2007) 125:244-255.

[9] Grasland B., Loizel C., Blanchard P., Oger A., Nignol A.C., Bigarré L., et al., Reproduction of PMWS in immunostimulated SPF piglets transfected with infectious cloned genomic DNA of type 2 porcine circovirus, Vet. Res. (2005) 36:685-697.

[10] Harms P.A., Sorden S.D., Halbur P.G., Bolin S.R., Lager K.M., Morozov I., Paul P.S., Experimental reproduction of severe disease in $\mathrm{CD} / \mathrm{CD}$ pigs concurrently infected with type 2 porcine circovirus and porcine reproductive and respiratory syndrome virus, Vet. Pathol. (2001) 38:528-539.

[11] Ihaka R., Gentleman R., R: a language for data analysis and graphics, J. Comp. Graph. Statist. (1996) 5:299-314

[12] Klinkenberg D., de Bree J., Laevens H., De Jong M.C., Within- and between-pen transmission of Classical Swine Fever Virus: a new method to estimate the basic reproduction ratio from transmission experiments, Epidemiol. Infect. (2002) 128:293299.

[13] Krakowka S., Ellis J.A., Meehan B., Kennedy S., Mcneilly F., Allan G., Viral wasting syndrome of swine: Experimental reproduction of postweaning multisystemic wasting syndrome in gnotobiotic swine by coinfection with porcine circovirus 2 and porcine parvovirus, Vet. Pathol. (2000) 37:254-263.
[14] Krakowka S., Ellis J.A., McNeilly F., Ringler S., Rings D.M., Allan G., Activation of the immune system is the pivotal event in the production of wasting disease in pigs infected with Porcine Circovirus-2 (PCV-2), Vet. Pathol. (2001) 38:31-42.

[15] Krakowka S., Ellis J., McNeilly F., Waldner C., Allan G., Features of porcine circovirus-2 disease: correlations between lesions, amount and distribution of virus, and clinical outcome, J. Vet. Diagn. Invest. (2005) 17:213-222.

[16] López-Soria S., Segalés J., Rose N., Viñas M.J., Blanchard P., Madec F., et al., An exploratory study on risk factors for postweaning multisystemic wasting syndrome (PMWS) in Spain, Prev. Vet. Med. (2005) 69:97-107.

[17] Madec F., Eveno E., Morvan P., Hamon L., Blanchard P., Cariolet R., et al., Post-weaning multisystemic wasting syndrome (PMWS) in pigs in France: clinical observations from follow-up studies on affected farms, Livest. Prod. Sci. (2000) 63:223-233.

[18] Magar R., Müller P., Larochelle R., Retrospective serological survey of antibodies to porcine circovirus type 1 and type 2, Can. J. Vet. Res. (2000) 64:184186.

[19] McCallum H., Barlow N., Hone J., How should pathogen transmission be modelled?, Trends Ecol. Evol. (2001) 16:295-300.

[20] McIntosh K.A., Harding J.C.S., Ellis J.A., Appleyard G.D., Detection of porcine circovirus type 2 viremia and seroconversion in naturally infected pigs in a farrow-to-finish barn, Can. J. Vet. Res. (2006) 70:58-61.

[21] McKeown N.E., Opriessnig T., Thomas P., Guenette D.K., Elvinger F., Fenaux M., et al., Effects of porcine circovirus type 2 (PCV2) maternal antibodies on experimental infection of piglets with PCV2, Clin. Diagn. Lab. Immunol. (2005) 12:13471351.

[22] Meerts P., Misinzo G., Lefebvre D., Nielsen J., Bøtner A., Kristensen C.S., Nauwynck H.J., Correlation between the presence of neutralizing antibodies against porcine circovirus 2 (PCV2) and protection against replication of the virus and development of PCV2-associated disease, BMC Vet. Res. (2006) 2:6.

[23] Rodríguez-Arrioja G.M., Segalés J., Calsamiglia M., Resendes A.R., Balasch M., PlanaDuran J., et al., Dynamics of porcine circovirus type 2 infection in a herd of pigs with postweaning multisystemic wasting syndrome, Am. J. Vet. Res. (2002) 63:354-357.

[24] Rodríguez-Arrioja G.M., Segalés J., Rosell C., Rovira A., Pujols J., Plana-Durán J., Domingo M., Retrospective study on porcine circovirus type 2 infection in pigs from 1985 to 1997 in Spain, J. 
Vet. Med. B Infect. Dis. Vet. Public Health (2003) 50: 99-101.

[25] Rose N., Larour G., Le Diguerher G., Eveno E., Jolly J.P., Blanchard P., et al., Risk factors for porcine post-weaning multisystemic wasting syndrome (PMWS) in 149 French farrow-to-finish herds, Prev. Vet. Med. (2003) 61:209-225.

[26] Rose N., Abhervé-Guéguen A., Le Diguerher G., Eveno E., Jolly J.P., Blanchard P., et al., Effect of the Pietrain breed used as terminal boar on Postweaning Multisystemic Wasting Syndrome (PMWS) in the offspring in four PMWS-affected farms, Livest. Prod. Sci. (2005) 95:177-186.

[27] SAS Institute Inc., SAS/STAT User's Guide, Version 9.1, SAS Institute, Cary, NC. USA, 2002.

[28] Segalés J., Domingo M., Postweaning multisystemic wasting syndrome (PMWS) in pigs. A review, Vet. Q. (2002) 24:109-124.

[29] Segalés J., Domingo M., Chianini F., Majó N., Domínguez J., Darwich L., Mateu E., Immunosuppression in postweaning multisystemic wasting syndrome affected pigs, Vet. Microbiol. (2004) 98:151-158

[30] Segalés J., Calsamiglia M., Olvera A., Sibila M., Badiella L., Domingo M., Quantification of porcine circovirus type 2 (PCV2) DNA in serum and tonsillar, nasal, tracheo-bronchial, urinary and faecal swabs of pigs with and without postweaning multisystemic wasting syndrome (PMWS), Vet. Microbiol. (2005) 111:223-229.

[31] Velthuis A.G., de Jong M.C., de Bree J., Nodelijk G., van Boven M., Quantification of transmission in one-to-one experiments, Epidemiol. Infect. (2002) $128: 193-204$

[32] Velthuis A.G., De Jong M.C., Kamp E.M., Stockhofe N., Verheijden J.H., Design and analysis of an Actinobacillus pleuropneumoniae transmission experiment, Prev. Vet. Med. (2003) 60:53-68.

[33] Woodbine K.A., Medley G.F., Slevin J., Kilbride A.L., Novell E.J., Turner M.J., et al., Risk factors for herd breakdown with Post-Weaning Multisystemic Wasting Syndrome (PMWS) in Great Britain, Pig J. (2006) 57. 